\title{
A cDNA library of the eutardigrade Hypsibius klebelsbergi Mihelčič, 1959 and analysis of the actin gene
}

\author{
Ernst KIEHL, Hieronymus DASTYCH ${ }^{1)}$, Jochen D'HAESE, and Hartmut GREVEN* \\ Institut für Zoomorphologie und Zellbiologie, Universität Düsseldorf, Universitätsstr,1, D-40225 Düsseldorf, Germany \\ ${ }^{1)}$ Biozentrum Grindel und Zoologisches Museum. Universität Hamburg, Martin-Luther-King-Platz 3, D-20146 Hamburg, Germany \\ *e-mail corresponding author: grevenh@uni-duesseldorf.de
}

\begin{abstract}
A cDNA library was constructed from the glacier-dwelling eutardigrade Hypsibius klebelsbergi from more than 2000 individuals collected in the Austrian Central Alps. RNA, DNA and proteins were successively isolated by the Trizol ${ }^{\mathbb{B}}$-method. From the RNA preparation a cDNA library was constructed with the cDNA inserted unidirectionally in the phagemid expression vector TriplEx2.

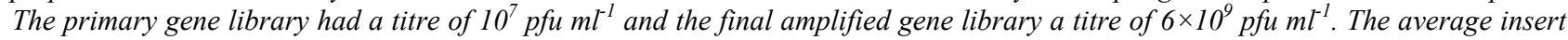
length was about $1.6 \mathrm{~kb}$. The partial sequence of $\mathrm{H}$. klebelsbergi actin $(746 \mathrm{bp}$ ) showed highest similarity to GenBank data of Drosophila melanogaster actin at the nucleic acid level (84.9\%) and at the amino acid level (98\%). Compared with actin fragments of the eutardigrades Ramazzottius oberhaeuseri $(450 \mathrm{bp}$ ) and Macrobiotus $\mathrm{sp}$. (453 bp) the identities were $85 \%$ - $81 \%$ and $100 \%$ $98 \%$ with respect to the nucleic/amino acids. Identity with actin fragments (359 bp) of Hypsibius dujardini from GenBank was $96 \%$ $100 \%$.
\end{abstract}

Key words: Eutardigrada, cDNA library, $m R N A$, actin

\section{INTRODUCTION}

Since 1996 a variety of molecular investigations using 18S rDNA have been published to elucidate phylogenetic and intergeneric relationships of Tardigrada (e.g. Garey et al. 1996, 1999; Giribet et al. 1996; Moon \& Kim 1996; Aguinaldo et al. 1997; Garey 2001; Jørgensen \& Kristensen 2004; Nichols et al. 2006; Kiehl et al. 2007, this issue). Only a few data exist on proteins derived from nucleic acid sequences (Regier et al. 2004; Schill et al. 2004).

A useful prerequisite for a detailed analysis of proteins is the construction of a cDNA library that allows the production of recombinant proteins. With respect to tardigrades only a single cDNAgene- library exists from the eutardigrade Hypsibius dujardini (Doyère, 1840) (TardiBase, Daub et al. 2003). The authors bred this species to obtain a sufficient number of specimens for RNA isolation and construction of an Expressed Sequence Tag (EST) library. From this library a considerable number of sequenced fragments of mRNAs have been incorporated in GenBank. For detailed protein analysis a cDNA expression library is necessary. However, tardigrades used for this purpose should be available in numbers of more than a thousand, they should be correctly identified to exclude species-specific protein variations, and contamination with foreign nucleic acids should be reduced to a minimum.

To overcome these difficulties we used the glacier dwelling eutardigrade Hypsibius klebelsbergi Mihelčič, 1959. This unique species lives in great numbers in cryoconite holes of several glaciers in the Alps. It can easily be collected and survives a long period at $2-4{ }^{\circ} \mathrm{C}$ when kept in water from the cryoconite holes supplemented occasionally by distilled water (Dastych et al. 2003). Due to the dark brown coloration of adults, $H$. klebelsbergi can be easily distinguished from other eutardigrades occasionally present in the same habitat (Dastych et al. 2003).

We report here on the construction of a cDNA library of $H$. klebelsbergi and on the sequence analysis of one actin isoform. Actin is one of the most highly conserved proteins and only its end terminal portions show some variability in isoforms. The actin filaments, part of the cytoskeleton of all eucaryotic cells, are formed by polymerization of monomer globular actin in muscles and non-muscle cells consisting of $374-376$ amino acids. The polymer state of actin is regulated by a great variety of actin binding proteins that probably vary among taxa (Kabsch \& Vandekerckhove 1992; Vandekerckhove 1993). The first results presented herein are part of a wider study that aims to characterise tardigrade actin and its isoforms and different actin binding proteins. Such findings will give new insights in functional adaptations and should enrich the discussion about the phylogenetic position of tardigrades.

\section{METHODS}

Hypsibius klebelsbergi was collected from cryoconite holes on the glacier Langtalferner, $2580 \mathrm{~m}$ a.s.1., in the Ötztal Alps (Austria). The animals were kept at approximately $4{ }^{\circ} \mathrm{C}$ until use. The water from the collecting site was occasionally complemented by frozen distilled water. 
Tab. 1. Data of the constructed cDNA library of Hypsibius klebelsbergi. pfu = plaque forming unit.

\begin{tabular}{ll}
\hline 1 - Titer of the primary gene library (volume: $500 \mu \mathrm{l})$ & $10^{7} \mathrm{pfu} \mathrm{ml}^{-1}$ \\
2 - Titer of amplified gene library (volume: $11 \mathrm{ml})$ & $6 \times 10^{9} \mathrm{pfu} \mathrm{ml}^{-1}$ \\
3 - Clones with insert & $>99 \%$ \\
4 - Insert length & $\sim 1.6 \mathrm{~kb}$ \\
5 - Vector & phagemid $\lambda$ TriplEx2 (Clontech) \\
6 - Selectable marker & Ampicillin resistance \\
7 - Host cells & E. coli XL1 blue \\
8 - Cloning site & SfiIA, SfilB \\
\hline
\end{tabular}

For construction of the cDNA library we used approximately 2000 animals. RNA, DNA and protein were successively isolated according to Chomczynski \& Sacchi (1987) using the commercial phenol mixture Trizol $^{\circledR}$ (GibcoBRL, Karlsruhe, Germany). In case of a limited sample size Trizol extraction allows a subsequent construction of a genomic library and the analysis of proteins. An extra reprecipitation of the RNA at the end of the procedure removed any remaining phenol traces. RNA and DNA were quantified spectrophotometrically (Beaven et al. 1955; Holtzhauer 1995). The protein-amount was calculated according to Warburg \& Christian (1941; see also Holtzhauer 1995).

Synthesis of complete cDNA (including complete 5' ends) and cDNA library construction were carried out following the instructions recommended by the manufacturers (BD Biosciences SMART cDNA library construction Kit, Clonetech, Palo Alto, USA, Gigapack ${ }^{\circledR}$ III Gold Packaging Extract, Stratagene, La Jolla, USA). According to the manufacturer, the resulting phage lambda gene library should contain the cDNA inserted into the expression vector $\lambda$ TriplEx 2 in the correct orientation for expression.

Actin fragments were amplified by using the primers Act U235 (AAYTGGGAYGAYATGGARAA) and Act L667 (GCCATYTCYTGYTCRAARTC) (Fretz \& Spindler 1999) and the general actin primers Actup (ATG GTN GGN ATG GGN CAR AAR) and Actdown (ATG GTN GGN ATG GGN CAR AAR) (Viray Master, Chicago, 1993). PCR was run with 15 min initial denaturation at $94{ }^{\circ} \mathrm{C}$ followed by 35 cycles of $30 \mathrm{sec}$, denaturation at $94{ }^{\circ} \mathrm{C}, 1 \mathrm{~min}$ annealing at $50{ }^{\circ} \mathrm{C}$, and 3 min elongation at $72{ }^{\circ} \mathrm{C}$. The program ended with 20 min incubation at $72{ }^{\circ} \mathrm{C}$. The sample volume was $25 \mu$. We used sample concentrations of $0.5 \mathrm{mM}$ each dNTP, $0.5 \mu \mathrm{M}$ each primer, $6 \mathrm{~m} \mathrm{M} \mathrm{Mg}^{++}, 1.25 \mathrm{U}$ Taq (Biomaster, Windeck, Germany) and $1 \mu \mathrm{l}$ cDNA gene library as template DNA in $1 \times$ PCR buffer (Biomaster, Windeck, Germany). PCR-products were separated in $1.2 \%$ agarose gels in TBE (90 mM Tris, $90 \mathrm{mM}$ boric acid, $2 \mathrm{mM}$ EDTA, $1 \mu \mathrm{g} \mathrm{ml}^{-1}$ ethidiumbromide) and photographed under UV-light. PCR-products were sequenced by Seqlab (Göttingen, Germany).

Sequences of $H$. klebelsbergi (Accession number AM 501548) were aligned with "Align plus 2.0" (Scientific \& Educational Software, Durham, USA), and compared with actin fragments obtained from $R$. ober- haeuseri (Accession number AM 501547), Macrobiotus sp. (Accession number AM 501546) (see Kaldeweier 2005), H. dujardini (Accession number CK 326228), Drosophila melanogaster (Meigen, 1930) actin5c (Accession number NCBI AE003435) (Adams et al. 2000).

\section{RESULTS AND DISCUSSION}

From about 2000 tardigrades carefully sorted out from the collection, we purified $17 \mu \mathrm{g}$ of RNA, $8.4 \mu \mathrm{g}$ of DNA and $302 \mu \mathrm{g}$ of protein. The construction of the cDNA gene library started with $1 \mu \mathrm{g}$ total RNA and resulted in a primary gene library with a titre of $107 \mathrm{pfu}$ $\mathrm{ml}^{-1}$ and a volume of $500 \mu \mathrm{l}$. The average insert length was about $1.6 \mathrm{~kb}$. The primary gene library was amplified starting with $5 \times 10^{5}$ potentially different clones. The titre of the final gene library was $6 \times 10^{9} \mathrm{pfu} \mathrm{ml}^{-1}$ in a total of $11 \mathrm{ml}$. Non-recombinant clones were below $1 \%$ (Tab. 1).

The titres of our primary and amplified gene library suggest that they consist of sufficient independent clones to represent most of the expressed genes of the animals. As only black-brown, i.e. more or less adult tardigrades were used, protein messages differentially expressed, for instance in various developmental stages or under special environmental conditions (e.g. freezing below or above habitat temperature) are absent.

We amplified parts of the actin sequence using the cDNA gene library as template for PCR. The various PCR products obtained by the combination of different primer pairs were sequenced and the sequences were combined. This resulted in a 746 bp long fragment of a H. klebelsbergi actin. Apparently this fragment represents the actin isoform, which is predominantly amplified under the experimental conditions used.

This sequence showed an $84.9 \%$ identity compared to the actin isoform ("Drome Act5c") of Drosophila melanogaster on the nucleic acid level and 98\% identity at the amino acid level. In the case of the five different amino acids, three of the hydrophobic amino acids were conservatively exchanged; alanin was exchanged by serine in two cases (Fig. 1). Comparing corresponding regions of the actin sequences of the eutardigrades $R$. oberhaeuseri (450 bp) and Macrobiotus sp. (453 bp), an $85 \%$ and $81 \%$ identity was detected at the gene level and a $100 \%$ and $98 \%$ identity at the amino acid level. Values for the eutardigrade $H$. dujardini (359 bp from the GenBank) were $96 \%$ and $100 \%$. 


\author{
Drome Act5c \\ Hypdu actin \\ Hypkl actin \\ Ramob actin \\ Macsp actin \\ Drome Act5c \\ Hypdu actin \\ Hypkl actin \\ Ramob actin \\ Macsp actin
}

Drome Act5c Hypdu actin

Hypkl actin

Ramob actin

Macsp actin

Drome Act5c

Hypdu actin

Hypkl actin

Ramob actin

Macsp actin

Drome Act5c Hypdu actin

Hypkl actin

Ramob actin

Macsp actin

Drome Act5c Hypdu actin Hypkl actin

Ramob actin

Macsp actin

Drome Act5c Hypdu actin

Hypkl actin

Ramob actin

Macsp actin

Drome Act5c Hypdu actin Hypkl actin

Ramob actin Macsp actin

Drome Act5c Hypdu actin Hypkl actin Ramob actin Macsp actin
AT GT GT GAC GAAGAA GT T GCT GCTCT GGTT GTCGACAACGGCTCT GGCAT GT GCAAGGCC ATG---GAAGACGAAGTTGCCGCCTT GGTCGTGGACAAT GGATCCGGTAT GT GCAAGGCC

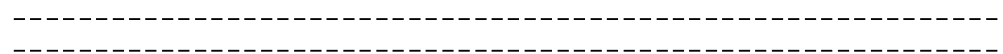

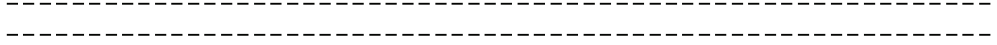

GGATTT GCCGGAGACGATGCTCCCCGCGCCGTCTTCCCATCGATTGTGGGACGTCCCCGT GGATTT GCCGGAGAT GACGCTCCCCGCGCCGTCTTCCCCTCCATCGTT GGCCGACCCCGT

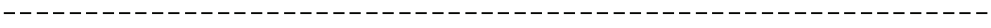

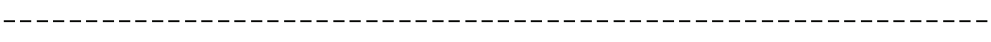

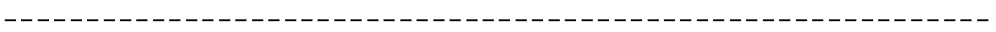

ATGGTNGGNATGGGNCARAA

CACCAGGGT GT GATGGTCGGCATGGGCCAGAA GGACTCGTACGTGGGT GATGAGGCGCAG CATCAGGGT GTCATGGTCGGTATGGGTCAAAAGGACAGCTACGTCGGTGATGAGGCCCAG

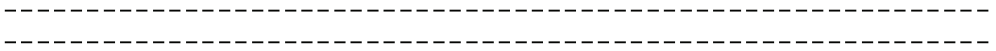

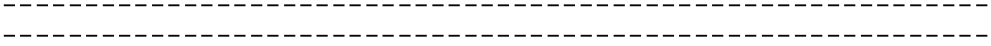

AAYTGG AGCAAGCGTGGTATCCTCACCCTGAAGTACCCCATTGAGCACGGTATCGTGACCAACTGG AGCAAGCGCGGTATCCTGACGCTCAAGTACCCCATCGAGCACGGCATCGTCACCAACTGG

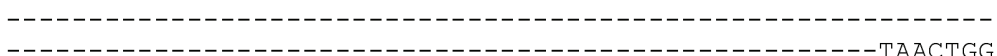

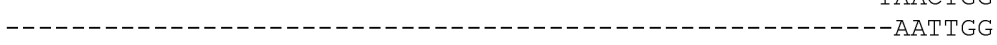

GAYGAYATGGARAA

GACGATATGGAGAA GATCT GGCACCACACCTTCTACAAT GAGCT GCGT GT GGCACCCGAG GATGACATGGAGAAGATCTGGCATCACACCTTCTACAACGAGCTCCGCGTGGCTCCCGAG --------GAGAAGATCT GGCATCATACCTTCTACAACGAGCTCCGCGTGGCACCCGAG GATGATATGGAAAAGATCT GGCATCATACCTTCTACAAT GAACTCCGT GTT GCCCCTGAG GATGATATGGAAAAGATCTGGCACCACACCTTCTACAACGAGCTCCGCGTGGCCCCCGAG

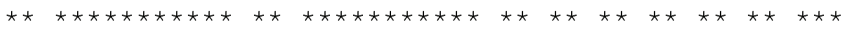

GAGCACCCCGTGCTGCTGACCGAGGCCCCGCTGAACCCCAAGGCCAACCGTGAGAAGATG GAACACCCCGTCCTCCTGACTGAGGCTCCCCTCAACCCCAAGGCCAACAGGGAAAAGATG GAGCACCCCGTCCTCCT GACTGAGGCTCCCCTCAACCCCAAGGCCAACAGGGAAAAGATG GAACACCCCGTCCTCCTCACTGAGGCCCCGCTCAACCCCAAGGCCAACAGAGAAAAGATG GAGCACCCCGTCCTGCTCACTGAGGCCCCCCTGAACCCCAAGGCCAATCGTGAGAAGATG

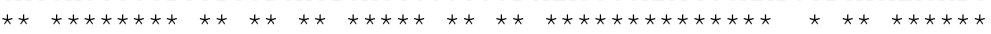

ACCCAGATCATGTTCGAGACCTTCAACACACCCGCCATGTATGTGGCCATCCAGGCTGTG ACCCAGATCATGTTCGAGACATTCAACACCCCCGCCATGTACGTCGCGATTCAGGCTGTG ACTCAGATCATGTTCGAGACATTCAACACCCCCGCCATGTACGTCGCGATTCAGGCTGTG ACCCAGATCATGTTCGAGACCTTCAACACCCCGGCCATGTACGTTGCCATCCAGGCCGTG ACCCAGATCATGTTCGAGACCTTCAACACCCCCGCCATGTACGTGGCCATCCAGGCTGTG

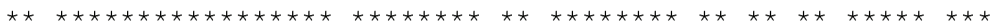

CTCTCGCTGTACGCCTCCGGTCGTACCACCGGTATCGTTCTGGACTCCGGCGATGGTGTC CTCTCCCTGTACGCGTCCGGTCGTACCACCGGTATCGTGCTGGACTCTGGTGATGGTGTC CTCTCCCTGTACGCGTCTGGTCGTACCACAGGTATCGTTCTGGATTCTGGTGATGGT GTC CT GTCCCTGTATGCGTCTGGTCGTACCACCGGTATCGTGCTGGACTCTGGTGATGGT GTC CT GTCCCTGTACGCCTCTGGTCGTACCACT GGTATCGTGTT GGATACT GGT GATGGT GTG

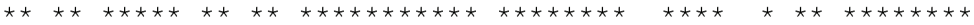

TCCCACACCGT GCCCATCTACGAGGGTTAT GCCCTTCCCCATGCCATCCTGCGTCTGGAT TCCCACACTGTCCCCATCTACGAAGGTTATGCTCT GCCTCACGCCATCCTCCGTCTGGAT TCCCACACT GTCCCGATCTACGAAGGTTAT GCTCT GCCCCATGCCATCCTCCGTCTGGAT AGCCACACT GT GCCCATCTATGAAGGTTAT GCTCT GCCCCATGCCATTCTCCGTCTGGAT TCTCACACTGTGCCCATCTACGAAGGCTATGCCCTGCCTCACGCCATCCTGCGTCTGGAT

Fig. 1. Comparison of the actin sequence of Drosophila melanogaster ("Drome Act5c") with actin fragments of the eutardigrades Hypsibius dujardini ("Hypdu actin"), Hysibius klebelsbergi ("Hypkl actin"), Ramazzottius oberhaeuseri ("Ramob actin") and Macrobiotus sp. ("Macsp actin"). An untranslated leader of the H. dujardini sequence was removed. The primers we used are underlined and written above the sequences at their correct positions. They are in 5' to 3' direction Actup, ActU235, ActL667 und Actdown. Matching positions are marked with a star. a: Nucleic acids. b: Amino acids. Continued. 
Drome Act $5 c$ Hypdu actin Hypkl actin Ramob actin Macsp actin

Drome Act5c Hypdu actin Hypkl actin Ramob actin Macsp actin

Drome Act5c Hypdu actin Hypkl actin Ramob actin Macsp actin

Drome Act5c Hypdu actin Hypkl actin Ramob actin Macsp actin

Drome Act5c Hypdu actin Hypkl actin Ramob actin Macsp actin

Drome Act5c Hypdu actin Hypkl actin Ramob actin Macsp actin

Drome Act5c Hypdu actin Hypkl actin Ramob actin Macsp actin

Drome Act $5 c$ Hypdu actin Hypkl actin Ramob actin Macsp actin

Drome Act5c Hypdu actin Hypkl actin Ramob actin Macsp actin

Drome Act5c Hypdu actin Hypkl actin Ramob actin Macsp actin
CT GGCT GGTCGCGATTT GACCGACTACCTGATGAAGATCCT GACCGAGCGCGGTTACTCT TTGGCCGGTCGCGACTTGACTGACTACTTGATGAAGATCCTGACTGAGCGCGG-TACAGC TTGGCCGGTCGCGACTTGACTGACTACTTGATGAAGATCCTGACTGAACGCGGTTACAGC TTGGCCGGTCGCGATCT GACTGACTATCTGATGAAGATTCT GACTGAACGTGGTTACAGC CT GGCT GGTCGCGATCT GACTGACTACCTCATGAAGGTCCTGACTGAGCGTGGCTACTCC

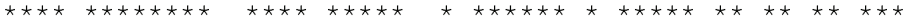

TTCACCACCACCGCTGAGCGTGAAATCGTCCGT GACATCAAGGAGAAGCT GT GCTAT GTT

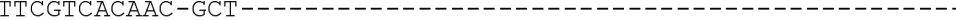
TTCGTCACAACCGCTGAACGAGAGATAGTCCGT GACATCAAGGAAAAGCTCT GCTACGTT TTCGTCACAACCGCT GAAC GCGAAATT GTCCGT GACATCAAGGAGAAACTCT GCTACGTC TTCACCACCACCGCCGAGCGCGAAATCGTCCGT GACATCAAGGA GAAGCTGT GCTACGTC

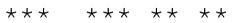

GAYTTYGARCARGARATGGC

GCCCTCGACTTTGAGCAGGAGATGGCCACCGCT GCCAGCAGCTCCTCGTTGGAGAAGTCC GCTCTCGACTTCGAGCAGGAAATGGCCACCGCT GCTGCATCCTCCTCCCTGGAAAAGAGC

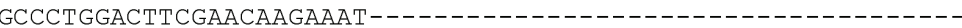
GCCCTTGACTTCGAACAAGAAATGGCA---

TACGAGCTGCCCGACGGACAGGTGATCACCATCGGCAACGAGCGTTTCCGCT GCCCCGAG TACGAACTTCCCGACGGTCAAGTCATCACCATCGGAAACGAGCGATTCCGAT GCCCTGAG -

GCCCTGTTCCAGCCCTCGTTCTTGGGAATGGAGGCTT GCGGCATCCACGAGACCACCTAC GCCCTG GCTCTCTTCCAACCCAGCTTTATCGGTATGGAGTCGT GCGGTATCCATGAGACCACCTAC

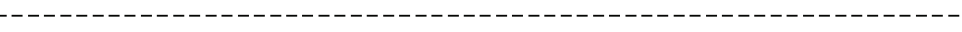

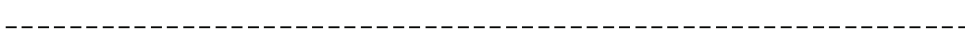

AACTCCATCATGAAGTGTGATGTGGATATCCGTAAGGATCT GTATGCCAACACCGTGCTG

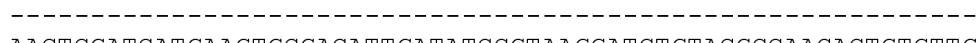
AACTCGATCATGAAGTGCGACATT GATATCCGTAAGGATCT GTACGCCAACACTGTGTTG -

TCCGGT GGCACCACCAT GTACCCT GGCATCGCCGACCGTAT GCAGAAGGAGATCACCGCC CCGGT GGCACCACCAT GT ACCCTGGCATCGCCGACCGTATGCAGA TCCGGCGGTACCACCAT GTACCCT GGTATT GCCGATCGCATGCAGAAGGAGATCACCGCC

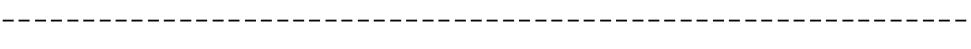

CTGGCACCGTCGACCAT GAAGATCAAGATCATT GCCCCGCCAGAGCGCAAGTACTCTGTC

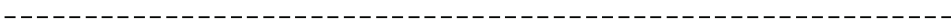

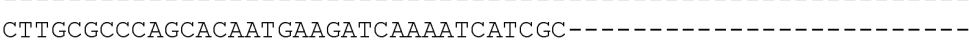

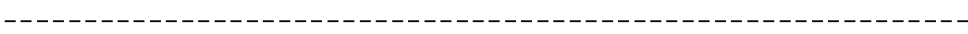
ACNTTYCARCARATGTGGATH TGGATCGGTGGCTCCATCCTGGCTTCGCTGTCCACCTTCCAGCAGATGTGGATCTCCAAG -

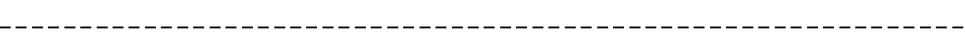

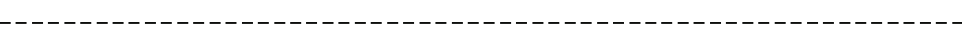
CAGGAGTACGACGAGTCCGGCCCCTCCATTGTGCACCGCAAGTGCTTCTAA - - - - - - - - - - - - - - - - - - - - - - - - - - - - - - - - - - - -

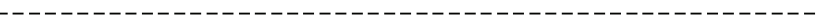

Fig. 1. Continuation. 


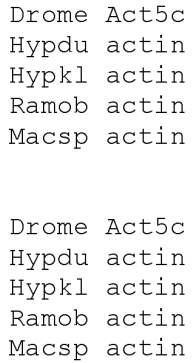

MCDEEVAALVVDNGS GMCKAGFAGDDAPRAVFP S IVGRP RHOGVMVGMGOKDSYVGDEAQ M-EDEVAALVVDNGS GMCKAGFAGDDAPRAVFP S IVGRPRHQGVMVGMGQKDSYVGDEAQ

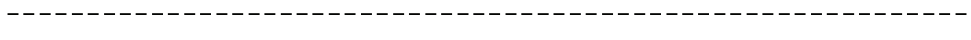

SKRGILTLKYPIEHG IVTNWDDMEKIWHHT FYNELRVAP EEHPVLLTEAPLNPKANREKM SKRGILTLKYP IEHG IVTNWDDMEKI WHHT FYNELRVAP EEHPVLLTEAP LNPKANREKM

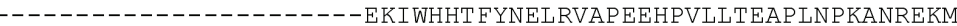
---------------NWDDME K I WHHT FYNEL RVAP EEHPVLLTEAP LNPKANREKM --------------NWDDMEKI WHHT FYNELRVAP EEHPVLLTEAPLNPKANREKM

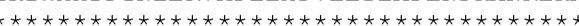

TQIMFETFNTPAMYVAIQAVLSLYASGRTTGIVLDSGDGVSHTVPI YEGYAL PHAILRLD TQIMFETFNTPAMYVAIQAVLSLYAS GRTT GIVLDSGDGVSHTVPIYEGYAL PHAILRLD TQIMFETENTPAMYVAIQAVLS LYAS GRTT GIVLDS GDGVSHTVP I YEGYAL PHA ILRLD TQIMFETFNTPAMYVA I OAVLSLYAS GRTT GIVLDS GDGVSHTVPI YEGYALPHAILRLD TQIMFETFNTPAMYVA I QAVLSLYAS GRTT GIVLDTGDGVSHTVPI YEGYAL PHA ILRLD

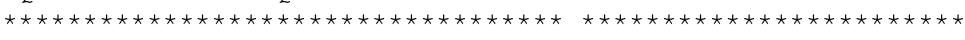

LAGRDLTDYLMKILTERGYSFTTTAERE IVRDI KEKLCYVALDFEQEMATAASSS SLEKS LAGRDLTDYLMKILTERG---TAS SQR------------------------LAGRDLTDYLMKILTERGYSFVTTAERE IVRDI KEKLCYVALDFEQEMATAAASS SLEKS LAGRDLTDYLMKILTERGYSFVTTAEREIVRDI KEKLCYVALDFEQE------------

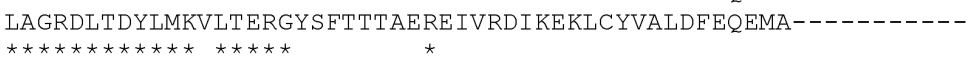

YELPDGQVIT I GNERFRCPEALFQPS FLGMEACGI HETT YNS IMKCDVDI RKDLYANTVL YELPDGQVIT I GNERFRCPEALFQPSFI GMESCGIHETTYNS IMKCDI DI RKDLYANTVL

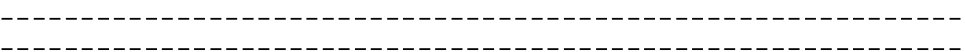
SGGTTMYPGIADRMQKE ITALAPSTMKIKI IAP PERKYSVWI GGS I LASLST FQQMWISK

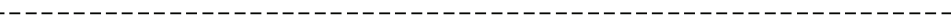

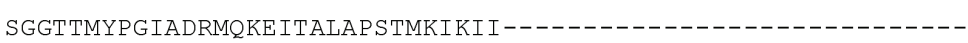

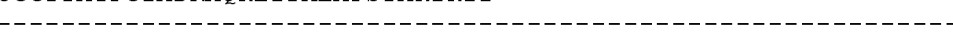
- - - - - -

QEYDES GPS IVHRKCF

- --------1

-

- - - - - -

Fig. 1. Continuation.

These differences appear rather high in view of the fact that actin exhibits $80.2 \%$ sequence conservation at the gene level between Homo sapiens and Saccharomyces cerevisiae Meyen \& Hansen, 1883, and 95\% conservation of the primary structure of the protein product (e.g. Vandekerckhove et al. 2003).

By screening our cDNA library with an actin fragment as a probe we have found eight different positive clones in the GenBank so far. Therefore, we expect to get the complete coding sequence of the gene as well as the $3^{\prime}$ and $5^{\prime}$ UTR of the predominant isoforms.

\section{REFERENCES}

Adams, M.D. et al. 2000. The genome sequence of Drosophila melanogaster. Science, 287: 2185-2195.
Aguinaldo, A.M., J.M. Tubeville, L.S. Linford, M.C. Rivera, J.R. Garey, R.A. Raff \& J.A. Lake. 1997. Evidence for a clade of nematodes, arthropods and other moulting animals. Nature, 387: 489-493.

Beaven, G.H., E.R. Holiday E.R. \& E.A. Johnson. 1955. Optical properties of nucleic acids and their components. In: E. Chargaff \& J.N. Davidson (Eds), The Nucleic Acids. Academic Press Inc., New York: 493- 554.

Chomczynski, P. \& N. Sacchi. 1987. Single-step method of RNA isolation by acid guanidinium thiocyanate-phenolchloroform extraction. Anal. Biochem., 162: 156-159.

Daub, J., F. Thomas, A. Aboobaker \& M.L. Blaxter. 2003. A survey of genes expressed in the tardigrade Hypsibius dujardini. See NCBI CK326773 and http://zeldia.cap. ed.ac.uk/tardigrades/tardibase.html.

Dastych, H., H.-J. Kraus \& K. Thaler. 2003. Redescription and notes on the biology of the glacier tardigrade Hypsibius klebelsbergi Mihelcic, 1959 (Tardigrada), based on 
material from the Ötztal Alps, Austria. Mitt. Hamb. Zool. Ms. Inst., 100: 73-100.

Fretz, A. \& K.D. Spindler. 1999. Hormonal regulation of actin and tubulin in an epithelial cell line from Chironomus tentans. Arch. Insect. Biochem. Physiol., 41: 71-78.

Garey, J.R. 2001. Ecdysozoa: The relationship between Cycloneuralia and Panarthropoda. Zool. Anz., 240: 321330.

Garey, J.R., M. Krotec, D.R. Nelson \& J. Brooks. 1996. Molecular analysis supports a tardigrade-arthropod association. Invertebr. Biol., 115: 79-88.

Garey, J.R., D.R. Nelson, L.M. Mackey \& J. Li. 1999. Tardigrade phylogeny: Congruence of morphological and molecular evidence. Zool. Anz., 238: 205-210.

Giribet, G., S. Carranza, J. Baguna, M. Riutort \& C. Ribeira. 1996. First molecular evidence for the existence of a tardigrada+arthropoda clade. Mol. Biol. Evol., 13: 76-84.

Holtzhauer, M. 1995. Biochemische Labormethoden. Springer Verlag, Berlin: $195 \mathrm{pp}$

Jørgensen, A. \& R.M. Kristensen. 2004. Molecular phylogeny of Tardigrada - investigation of the monophyly of Heterotardigrada. Mol. Phylogen. Evol., 32: 666-670.

Kabsch, W. \& J.S. Vanderkerckhove. 1992. Structure and function of actin. Ann. Rev. Biophys. Biomol. Struct., 21: 49-76.

Kaldeweier, A. 2005. Molekularbiologische Untersuchungen zur Verbreitung und Variabilität von Aktin und aktinbindenden Proteinen bei ausgewählten Taxa (Ecdysozoa und
Nicht-Ecdysozoa). Diplomarbeit am Institut für Zoologie II der Universität Düsseldorf.

Kiehl, E., H., Dastych, J., D'Haese \& H. Greven. 2007. The 18S rDNA sequences support polyphyly of the Hypsibiidae (Eutardigrada). In: G. Pilato \& L. Rebecchi (Eds), Proceedings of the Tenth International Symposium on Tardigrada. J. Limnol., 66(Suppl. 1): 21-25.

Moon, S. \& W. Kim. 1996. Phylogenetic position of the Tardigrada based on the $18 \mathrm{~S}$ ribosomal RNA gene sequences. Zool. J. Linn. Soc., 116: 61-69

Nichols, P.B., D.R. Nelson \& J.R. Garey. 2006. A family level analysis of tardigrade phylogeny. Hydrobiologia, 558: 5360.

Regier, J.C., J.W. Shultz, R.E. Kambic \& D.R. Nelson. 2004. Robust support for tardigrade clades and their ages from three protein-coding nuclear genes. Invertebr. Biol., 123: 93-100.

Schill, R.O., G. Steinbrueck \& H.-R. Koehler. 2004. Stress gene (hsp70) sequences and quantitative expression in Milnesium tardigradum (Tardigrada) during active and cryptobiotic stages. J. Exp. Biol., 207: 1607-1613.

Vandekerckhove, J.S. 1993. Actins. In: T. Kreis \& R. Vale (Eds), Guidebook to the cytoskeletal and motor proteins. Oxford University Press Inc., New York: 13-15.

Viraj Master, Chicago. 1993. http://www.bio.net/hypermail/ /immuno/1993-September/000528.html

Warburg, O. \& W. Christian. 1941. Isolierung und Kristallisation des Gärungsferments Enolase. Biochem. Z., 310: 384-421. 